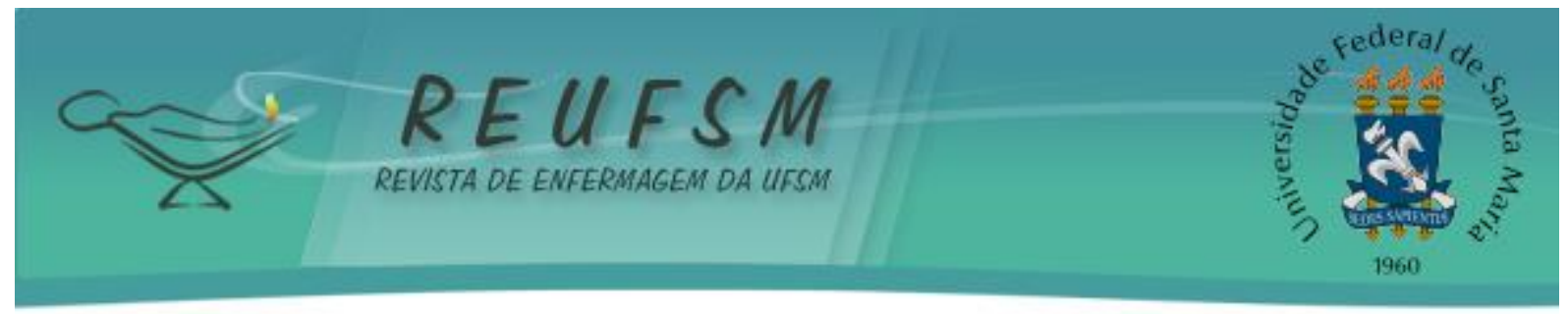

ARTIGO ORIGINAL

\title{
CARACTERÍSTICAS DEMOGRÁFICAS, SOCIAIS E CLÍNICAS DE MULHERES EM IDADE FÉRTIL ATINGIDAS PELA HANSENÍASE
}

\section{DEMOGRAPHIC, SOCIAL AND CLINICAL CHARACTERISTICS OF WOMEN IN CHILDBEARING AGE AFFECTED BY LEPROSY}

\section{CARACTERÍSTICAS DEMOGRÁFICAS, SOCIALES Y CLÍNICAS DE MUJERES EN EDAD FÉRTIL QUE SUFREN DE LA ENFERMEDAD DE HANSEN}

\author{
Clodis Maria Tavares ${ }^{1}$ \\ Tâmyssa Simões dos Santos ${ }^{2}$ \\ Nataly Mayara Cavalcante Gomes ${ }^{3}$ \\ Reinaldo Antônio Silva-Sobrinho ${ }^{4}$ \\ Fernanda Silva Goes ${ }^{5}$ \\ Ana Maria de Almeida ${ }^{6}$
}

Doi: $10.5902 / 2179769230173$

RESUMO: Objetivo: descrever as características demográficas, sociais e clínicas de mulheres em idade fértil atingidas pela hanseníase em uma capital do nordeste brasileiro. Método: estudo descritivo de corte transversal, com abordagem quantitativa. A população do estudo foi composta por 217 mulheres, e resultou em uma amostra intencional de 60, que atenderam aos critérios de inclusão. Resultados: os dados revelaram que 95,0\% do conjunto amostral, encontrava-se na faixa de 20 a 49 anos. O modo de detecção que apresentaram uma maior porcentagem foram: o encaminhamento e a demanda espontânea, constituindo 60,0\% dos casos. Identificou-se que 30,0\% foram diagnosticadas na forma dimorfa, o que afirma os dados referente à classificação multibacilar. Conclusão: as características demográficas, sociais e clínicas foram: mulheres economicamente ativas, predominantemente analfabetas, que possuem companheiros e desempenham ocupações remuneradas e domésticas.

Descritores: Hanseníase; Doenças negligenciadas; Características da população; Saúde da mulher

ABSTRACT: Aim: to describe demographic, social and clinical characteristics of women in childbearing age affected by leprosy in a capital city in the northeast of Brazil. Method: crosssectional descriptive study, with quantitative approach. The study was composed by 217 women, resulting in an intentional sample of 60, who have attended the criteria of inclusion. Results: the

\footnotetext{
1 Enfermeira. Doutora em Enfermagem pela Universidade de São Paulo - USP. Professora titular da Universidade Federal de Alagoas - UFAL. Maceió/Alagoas, Brasil. E-mail: clodistavares@yahoo.com.br

${ }^{2}$ Enfermeira. Mestre em Educação em Ciências e Saúde pela Universidade Federal do Rio de Janeiro -UFRJ. Professora do Centro Universitário Mauricio de Nassau - UNINASSAU. Maceió/Alagoas, Brasil. E-mail: simoestamyssa@gmail.com

${ }^{3}$ Acadêmica de Enfermagem pela Escola de Enfermagem e Farmácia da Universidade Federal de Alagoas UFAL. Maceió/Alagoas, Brasil. E-mail: natallymayara@ hotmail.com

${ }^{4}$ Enfermeiro. Pós-Doutorado em Saúde Pública pela Universidade de São Paulo - USP. Professor Adjunto C do Curso de Enfermagem pela Universidade Estadual do Oeste do Paraná - UNIOESTE. Curitiba/Paraná, Brasil. Email: reinaldo.sobrinho@unioeste.br

${ }^{5}$ Enfermeira. Graduação pela Universidade Federal de Alagoas - UFAL. Enfermeira da Prefeitura Municipal de Porto de Pedras/Alagoas, Brasil. E-mail: nandinhagoes_@hotmail.com

${ }^{6}$ Enfermeira. Livre-docência e Doutorado em Enfermagem pela Universidade de São Paulo, USP, Brasil. Senior and Associate Professor University of São Paulo at Ribeirão Preto College of Nursing Department of MaternalInfant and Public Health Nursing. Ribeirão Preto/São Paulo, Brasil. E-mail: ama2754@ gmail.com
} 


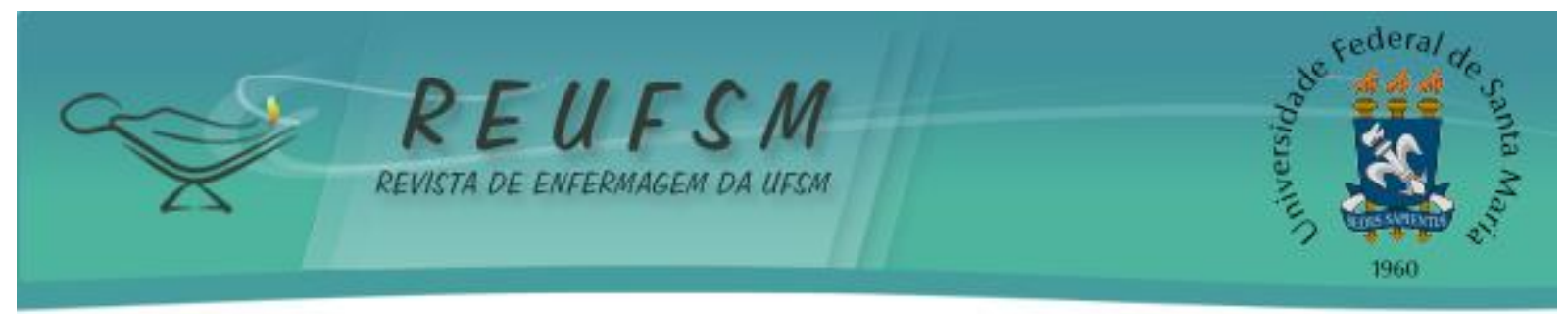

data showed that $95 \%$ of the subjects were set between 20 and 49 years of age. The detection modes which presented a higher percentage were both the handling and the spontaneous demands, with $60 \%$ of the cases. It was identified that $30 \%$ were diagnosed in the dimorphic form, which is confirmed by the data regarding the multibacillary classification. Conclusion: the demographic, social and clinical characteristics were: economically active women, mostly illiterate, who are married and perform both remunerated and domestic occupations.

Descriptors: Leprosy; Neglected diseases; Population characteristics; Women's health

RESUMEN: Objetivo: describir las características demográficas, sociales y clínicas de mujeres en edad fértil que sufren de la enfermedad de Hansen en una capital del Nordeste brasileño. Método: estudio descriptivo de cohorte transversal, con análisis cuantitativo. La población del estudio fue compuesta por 217 mujeres, de esas se estableció una muestra intencional de 60, en las cuales se verificó los criterios de inclusión. Resultados: los dados evidenciaron que 95,0\% del conjunto de la muestra tenía entre 20 y 49 años. El modo de detección que presentó un mayor porcentaje fue: la orientación y búsqueda de tratamiento espontánea, constituyendo $60 \%$ de los casos. También se identificó que $30 \%$ de esas mujeres fue diagnosticada de forma dimorfa, lo que ratifica los dados sobre la clasificación multibacilar. Conclusión: las características demográficas, sociales y clínicas de ese grupo fue: mujeres económicamente activas, en la mayoría analfabetas, que tienen compañeros y desempeñan ocupaciones remuneradas y domésticas.

Descriptores: Lepra; Enfermedades desatendidas; Características de la población; Salud de la mujer

\section{INTRODUÇÃO}

A hanseníase apresenta tendência crescente em países subdesenvolvidos e em desenvolvimento, o que representa um desafio às precárias condições da Saúde Pública. A forma de transmissão, assim como o agravamento dos casos, está diretamente relacionada às condições socioeconômicas da população, por estar atrelada à aglomeração de pessoas, que facilita sua transmissão. ${ }^{1-2}$

O boletim epidemiológico da Organização Mundial de Saúde (OMS) aponta que a hanseníase está presente em 25 dos 35 países que compõem o Continente Americano, e todos, com exceção do Brasil, já eliminaram a doença como um problema de saúde pública (menos de um caso por cada 10.000 habitantes) em nível nacional. ${ }^{2-3}$

A Índia é o país que apresenta o primeiro lugar em número absoluto no mundo, seguida pelo Brasil. Em 2015, foram diagnosticados 210.758 casos novos no mundo, sendo 127.326 na Índia e 26.395 no Brasil, dos quais, 7,5\% já apresentavam grau II de incapacidade. Esta situação afeta a qualidade de vida de milhares de pessoas, uma vez que a doença compromete o sistema 


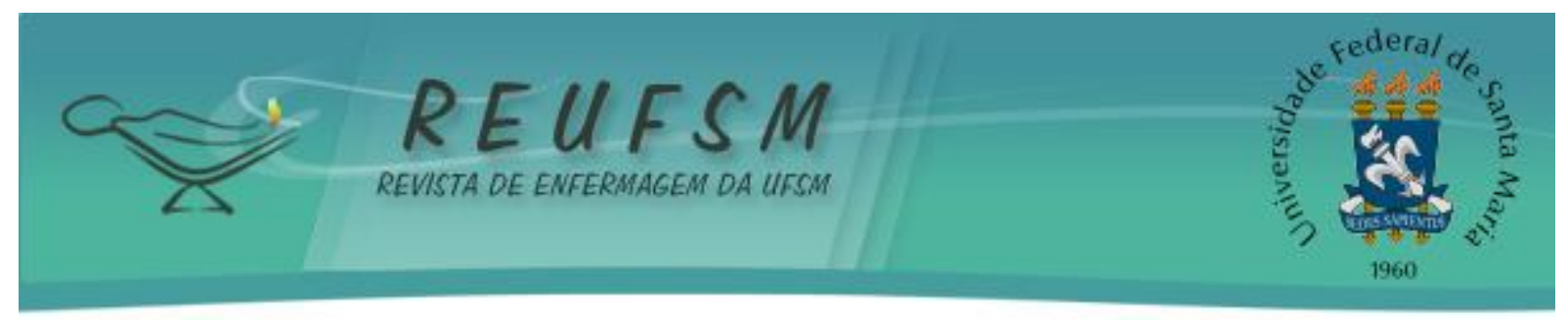

imunológico, a nocicepção, a visão e o tato, tornando-os mais vulneráveis a diversos riscos, tais como: acidentes, queimaduras, feridas, infecções, amputações, entre outros. ${ }^{4-5}$

No Brasil, houve uma redução na prevalência nos últimos anos, passando de 4,52/10 mil habitantes em 2003, para 1,01 em 2015. Entretanto, a taxa de detecção geral foi de 14,06/100 mil habitantes, correspondendo a 28.761 casos novos da doença no país. As áreas de maior risco de adoecimento estão concentradas nas regiões Norte, Nordeste e Centro-Oeste do país. ${ }^{1-2,6}$

Dados epidemiológicos de Alagoas apontaram que, em 2015, foi alcançado um coeficiente de detecção de 10,57/100.000 habitantes. Considerando-se os parâmetros da OMS e do Ministério da Saúde (MS), o período exibe uma alta taxa de detecção. Na avaliação nacional do MS, o estado ocupa o $19^{\circ}$ lugar no Brasil, em número de casos. Um fato preocupante é que os casos novos têm ocorrido em pessoas cada vez mais jovens, o que requer ações voltadas para este grupo, a fim de se obter a prevenção das incapacidades físicas. ${ }^{6}$

A abordagem dada às mulheres atingidas pela hanseníase na faixa etária reprodutiva deve-se às alterações hormonais e aos fatores emocionais (medo, depressão, ansiedade), que podem contribuir com a evolução da doença e surgimento dos eventos reacionais. Isso fortalece, as modificações que ocorrem no corpo da mulher que foi acometida pelo bacilo de Hansen, o que repercute em sua imagem. Desta forma, estas necessitam de atenção por parte dos profissionais de saúde, visto que, esta enfermidade se não for diagnosticada e tratada precocemente leva as incapacidades físicas. ${ }^{7-8}$

Diante dos fatos, o presente estudo teve como questão norteadora: quais as características demográficas, sociais e clínicas de mulheres em idade fértil atingidas pela hanseníase em uma capital do nordeste brasileiro? As pesquisas vêm apontando um maior número de casos em mulheres na faixa etária reprodutiva. Tal dado, pode ser identificado por meio de estudos realizados em Recife, Fortaleza, Rondônia, que trataram que a maioria das entrevistadas possuíam em torno de 20 a 40 anos de idade. ${ }^{9-11}$

Desta maneira, investigar esta população trará informações que subsidiarão o planejamento de ações e intervenções que possibilitem a estas mulheres a utilização de proteção efetiva no sentido de prevenir complicações decorrentes da evolução da doença e manifestações reacionais. 


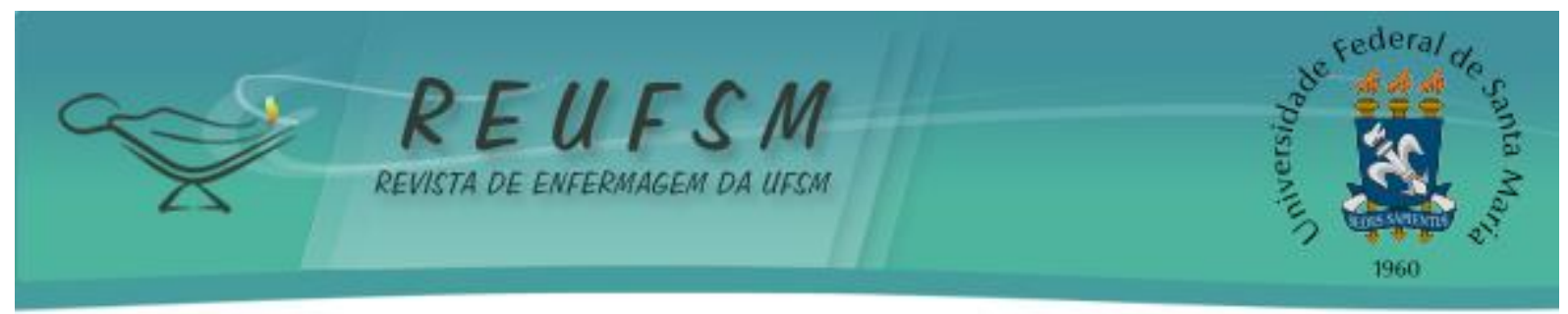

Em vista disso, o objetivo do trabalho foi descrever as características demográficas, sociais e clínicas de mulheres em idade fértil atingidas pela hanseníase em uma capital do nordeste brasileiro.

\section{MÉTODO}

Trata-se de estudo descritivo de corte transversal, com abordagem quantitativa realizado em 14 Unidades Básicas de Saúde (UBS), localizadas no município de Maceió, Alagoas, Brasil. A população do estudo foi composta por 217 mulheres, que estavam registradas no Sistema de informações de agravos de notificação (SINAN) e cadastradas nos livros das UBS. A amostra intencional foi constituída por 60 mulheres que atenderam aos critérios de inclusão.

Para seleção da amostra foram utilizados os seguintes critérios de inclusão: mulheres diagnosticadas com hanseníase entre o período de 2006-2011; com idade compreendida entre 10-49anos (no período selecionado não havia casos registrados de menores de 14 anos, desta forma, a idade compreendida passou a ser de 14-49anos); e residentes em Maceió, Alagoas, Brasil. Quanto ao critério de exclusão: mulheres que não foram encontradas por razões diversas tais como: mudanças de bairro, município ou estado; telefones e endereços desatualizados; ou evoluíram para óbito.

A coleta de dados foi realizada no período de janeiro a abril de 2013. Os encontros foram agendados previamente nas UBS. Para as participantes que não puderam comparecer, prosseguiu-se com contato telefônico, com intuito de agendar as visitas em seus domicílios com a ajuda dos Agentes Comunitários de Saúde (ACS). Após a coleta de dados com instrumento de pesquisa, foram utilizados os prontuários das mulheres em idade fértil atingidas pela hanseníase, para o levantamento dos dados clínicos.

As variáveis envolvidas no estudo compreenderam: idade (em anos completos) no diagnóstico da hanseníase, ter companheiro, escolaridade, ocupação, religião, forma clínica da doença, classificação operacional, resultado de baciloscopia, número de pessoas na família (que coabitam o mesmo lar), número de contatos registrados, número de contatos examinados, grau de incapacidade física no momento do diagnóstico e na alta por cura, número de lesões cutâneas, modo de detecção e entrada, e reações hansênicas (antes do diagnóstico, durante e após o tratamento). 


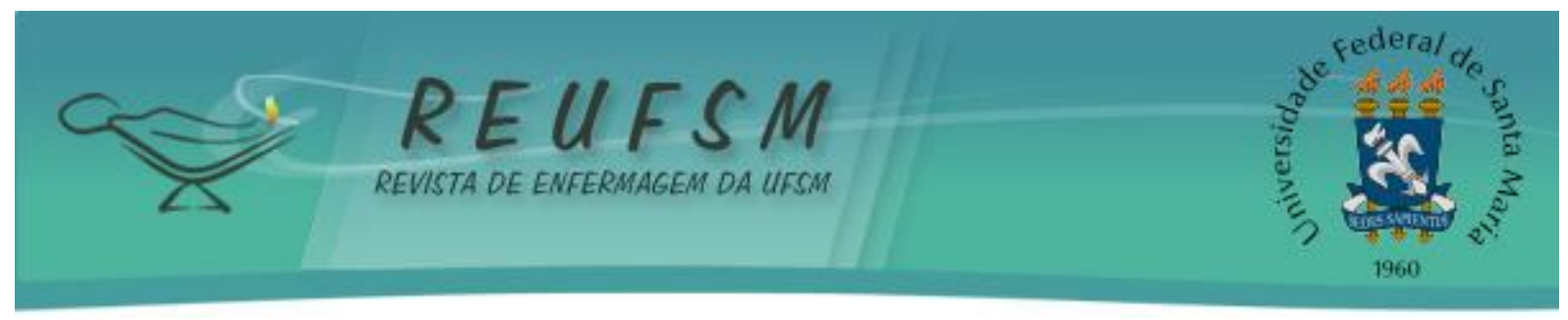

Na sequência, os dados foram copilados e realizada digitação dupla, por pesquisadores distintos. Foi construído e armazenado em uma planilha do Microsoft Office Excel® para a codificação das variáveis, e seguiu-se com a validação das planilhas. Após esta etapa, os dados foram importados e processados pelo programa Statistical Package for the Social Sciences (SPSS) versão 17.0 para Windows. As análises descritivas foram realizadas visando caracterizar a população do estudo.

Buscando zelar pela integridade ética dos participantes envolvidos no estudo, e com base nas diretrizes regulamentadoras das pesquisas com seres humanos contidas na resolução 466/2012. Todos os participantes foram informados sobre a finalidade, objetivos e os métodos do estudo por meio do Termo de Consentimento Livre e Esclarecido (TCLE). Foram elaborados dois TCLE, um para as mulheres acima de 18 anos, e outro para os responsáveis pelas mulheres menores de 18 anos.

O presente estudo foi apreciado e aprovado pelo Comitê de Ética em Pesquisa da Faculdade de Ciências Biológicas e da Saúde do Centro Universitário de Maceió (CESMAC), sob protocolo de pesquisa $\mathrm{n}^{\mathrm{o}}$ 1291/12.

\section{RESULTADOS}

Participaram do estudo 60 mulheres atingidas pela hanseníase. Os dados revelaram que $95,0 \%$ do conjunto amostral, encontrava-se na faixa de 20 a 49 anos. Considerando a escolaridade, observou-se a predominância de mulheres com baixo nível educacional. Em relação à ocupação, 55,0\% delas desempenhavam ocupações remuneradas, incluindo marisqueiras, empregadas domésticas, rendeiras, diaristas, entre outras.

Tabela 1 - Distribuição das mulheres em idade fértil atingidas pela hanseníase, segundo características sociodemográficas. Maceió-Alagoas, 2006 a 2011 (n=60).

\begin{tabular}{lcc}
\hline Variáveis & $\mathbf{N}$ & Distribuição \\
\cline { 2 - 3 } & & \% \\
\hline Idade & 3 & 5,0 \\
$14-19$ & 12 & 20,0 \\
$20-29$ & 45 & 75,0 \\
$30-49$ & & \\
Companheiro & 42 & 70,0 \\
Sim & 17 & 28,3 \\
Não & 1 & 1,7 \\
Não respondeu & &
\end{tabular}




$\begin{array}{lcc} & \\ \text { Analfabeto/Fundamental Incompleto } & 32 & 53,3 \\ \text { Fundamental Completo Médio Incompleto } & 13 & 21,7 \\ \text { Médio Completo } & 12 & 20,0 \\ \text { Superior Completo } & 3 & 5,0 \\ \text { Religião } & 37 & 61,6 \\ \text { Católica } & 16 & 26,7 \\ \text { Evangélica } & 1 & 1,7 \\ \text { Espírita } & 6 & 10,0 \\ \text { Não tem religião } & & \\ \text { Ocupação } & 27 & 45,0 \\ \text { Do lar } & 33 & 55,0 \\ \text { Ocupações remuneradas } & 22 & 36,7 \\ \text { Número de pessoas na família } & 21 & 35,0 \\ \text { 1 a 3 } & 17 & 28,3 \\ \text { 4 a 5 } & & \\ \text { 6 a 14 } & \end{array}$

$\mathrm{Na}$ Tabela 2, observa-se que o modo de detecção que apresentaram uma maior porcentagem foram: o encaminhamento e a demanda espontânea, constituindo 60,0\% dos casos.

Tabela 2 - Distribuição das mulheres em idade fértil atingidas pela hanseníase, segundo modo de detecção e modo de entrada no sistema de saúde, Maceió-Alagoas, 2006 a 2011 (n=60).

\begin{tabular}{lcc}
\hline Variáveis & N & Distribuição \\
\cline { 2 - 3 } & & $\%$ \\
Modo de deteç̃ão $^{1}$ & 18 & 30,0 \\
Encaminhamento $^{\text {Demanda espontânea }}$ & 18 & 30,0 \\
Exame de coletividade & 2 & 3,3 \\
Exame de contatos & 1 & 1,7 \\
Não informado & 21 & 35,0 \\
& & \\
Modo de entrada & & \\
Caso novo & & 73,3 \\
Transferência & 44 & 16,8 \\
Recidiva & 10 & 3,3 \\
Reingresso & 2 & 3,3 \\
Não informado & 2 & 3,3 \\
\hline Nor
\end{tabular}

Nota 1 - Modo de detecção: é a informação que é dada na ficha de notificação compulsória que informa como caso foi identificado para o diagnóstico da Hanseníase.

Nota 2 - Modo de entrada: é a informação que é dada na ficha de notificação compulsória que identifica como o caso deu entrada para o diagnóstico de hanseníase.

A tabela 3 descreve a distribuição das formas clínicas, classificação operacional e os números de lesões das mulheres atingidas pela hanseníase. Identificou-se que 30,0\% foram diagnosticadas na forma dimorfa, o que afirma os dados referente à classificação multibacilar. 


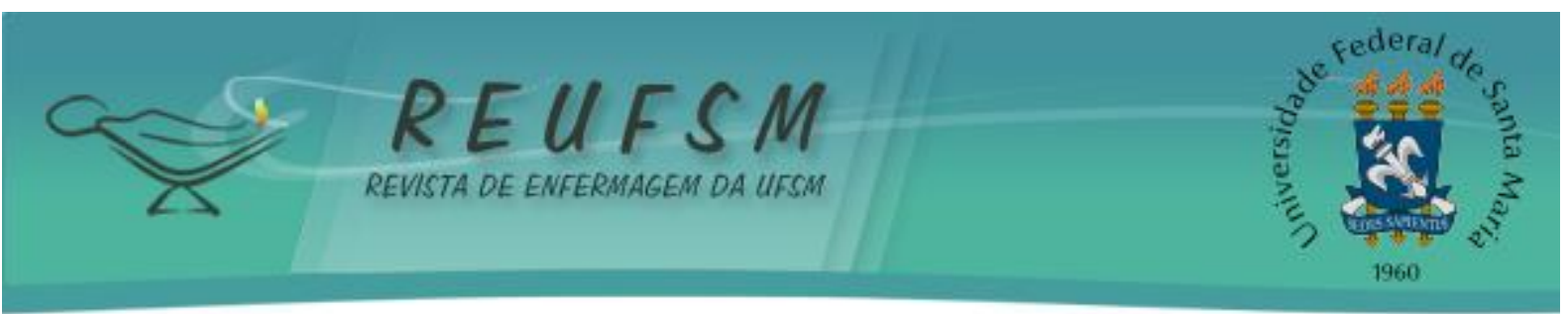

É relevante apontar que, $81,7 \%$ dos casos não tinham exame de baciloscopia registrado no boletim de acompanhamento de hanseníase ou no prontuário.

Tabela 3 - Distribuição das mulheres em idade fértil atingidas pela hanseníase, segundo forma clínica, classificação operacional, baciloscopia e número de lesões, Maceió-Alagoas, 2006 a $2011(n=60)$.

\begin{tabular}{lcc}
\hline Variáveis & \multicolumn{2}{c}{ Distribuição } \\
\cline { 2 - 3 } Forma Clínica & $\mathbf{N}$ & \% \\
\cline { 2 - 3 } Indeterminada & 9 & 15,0 \\
Tuberculóide & 12 & 20,0 \\
Dimorfa & 18 & 30,0 \\
Virchowiana & 6 & 10,0 \\
Não classificada & 15 & 25,0 \\
Classificação Operacional & & \\
Paucibacilar & 25 & 41,6 \\
Multibacilar & 34 & 56,7 \\
Não classificado & 1 & 1,7 \\
Número de lesões & & 10,0 \\
0 & 6 & 48,4 \\
1 a 5 & 29 & 41,6 \\
6 e mais & 25 & \\
\hline
\end{tabular}

Na tabela 4 os resultados apontam a hanseníase como um problema de saúde pública, uma vez que, no momento do diagnóstico 33,3\% das mulheres avaliadas, já apresentavam incapacidades físicas. É relevante informar que 46,7\% do total das entrevistadas, não foram submetidas à Avaliação Neurológica Simplificada no momento da alta.

Ademais, observa-se que $50,0 \%$ das pessoas avaliadas não tiveram seus contatos examinados, sendo que 55,0\% possuíam de uma a cinco pessoas da família registradas como contatos domiciliares. Evidencia-se também, que 66,6\% das mulheres avaliadas apresentaram reações hansênicas.

Tabela 4 - Distribuição das mulheres em idade fértil atingidas pela hanseníase, segundo o grau de incapacidade física no diagnóstico e na alta, número de contatos registrados, contatos examinados no momento do diagnóstico e reações hansênicas, Maceió-Alagoas, 2006 a 2011 (n=60).

\begin{tabular}{lcc}
\hline Variáveis & \multicolumn{2}{c}{ Distribuição } \\
\cline { 2 - 3 } Grau de incapacidade física no diagnóstico & $\mathbf{N}$ & \% \\
0 (Zero) & 32 & 53,4 \\
1 (Um) & 18 & 30,0 \\
2 (Dois) & 2 & 3,3 \\
Não avaliado & 5 & 8,3 \\
Não informado & 3 & 5,0
\end{tabular}




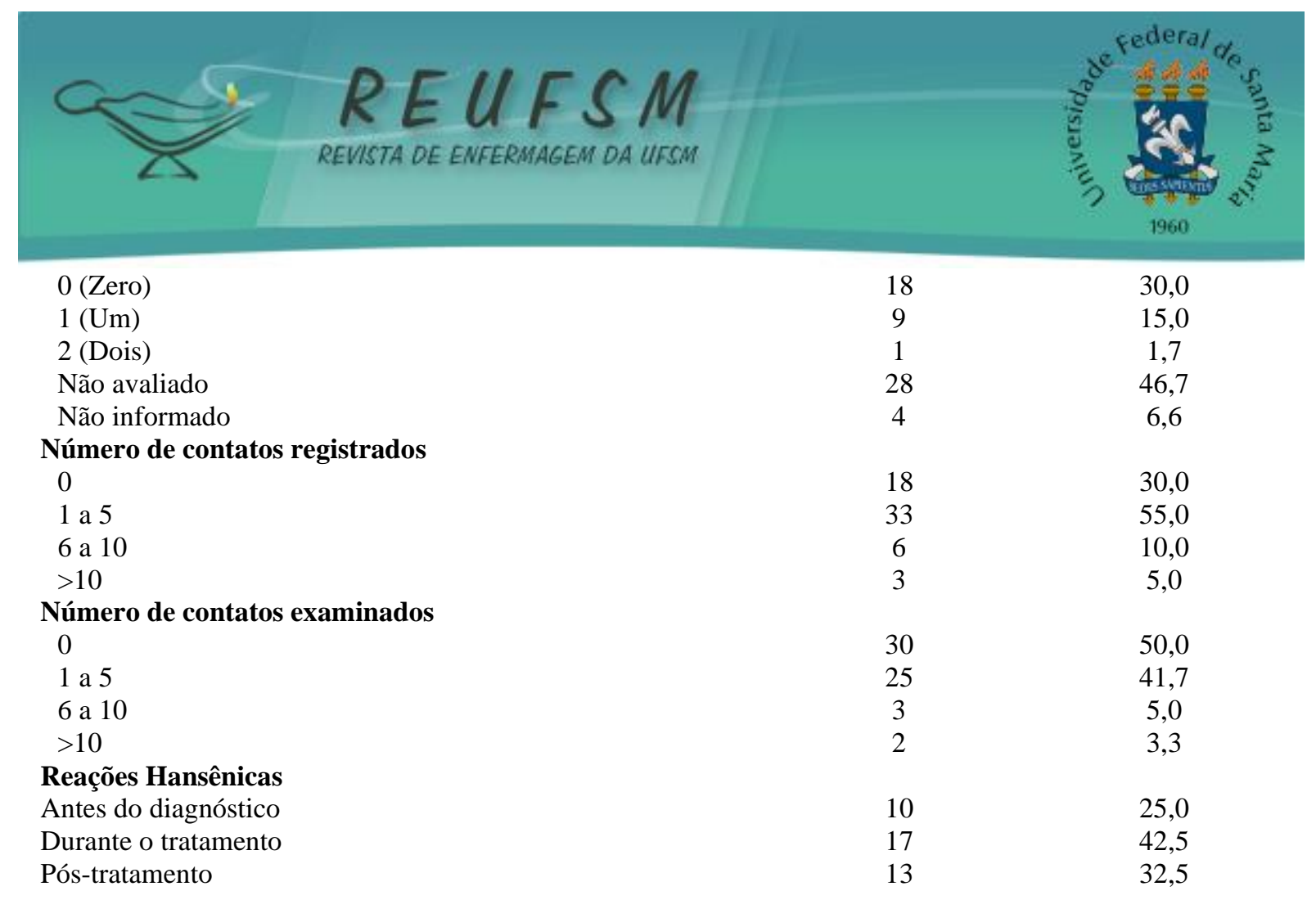

\section{DISCUSSÃO}

Com base no panorama atual da hanseníase, percebe-se que a redução desta doença no país representa um desafio para os serviços e profissionais da saúde. Neste cenário, ressalta-se o papel do enfermeiro no âmbito da atenção básica, no tocante as ações estratégicas para eliminação e controle das doenças infectocontagiosas. ${ }^{2}$

Ao tratar sobre o gênero, torna-se importante investigar o sexo feminino, em virtude das diversas alterações que ocorrem no corpo da mulher (puberdade, gestação, puerpério e menopausa) durante seu ciclo de vida, que podem estimular o desenvolvimento da doença. ${ }^{7-12}$ Diante destas informações, evidencia-se que todas as fases da vida das mulheres atingidas pela hanseníase tem seu grau de importância, no entanto, autores vem relatando que a faixa etária economicamente ativa pode trazer repercussões no ambiente de trabalho, devido a necessidade de adaptação, perda da produtividade, discussões sobre direitos trabalhistas, problemas com a autoimagem que dificultam a vida social. ${ }^{13-14}$

Neste sentido, ao analisar os resultados, pode-se observar que a faixa etária com maior número de casos foi de 30 a 49 anos. De maneira semelhante, um estudo realizado no Estado da Paraíba, apontou uma média de 43 anos. ${ }^{15}$ Este dado também é encontrado em pesquisa realizada em Recife, com mulheres privadas de liberdade, a qual identificou 74,8\% dos casos com faixa etária de 20 a 40 anos de idade. ${ }^{9}$ 


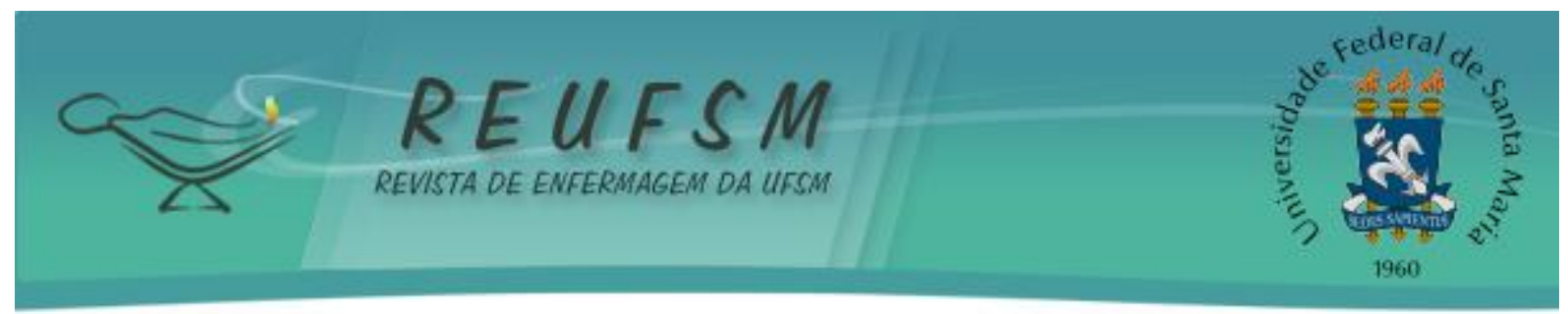

Apesar de a doença afetar pessoas de todas as idades e de ambos os sexos, as condições individuais, a alta endemicidade e condições socioeconômicas desfavoráveis, tais como, viver em locais insalubres e residências pequenas com alta densidade de pessoas, tem influenciado o aumento do risco de infecção e adoecimento. ${ }^{17}$ Tais fatores associados ao baixo nível escolar, além de estar associado ao elevado número de casos, corrobora com o diagnóstico tardio, por falta de conhecimento da população quanto as manifestações clínicas da doença. ${ }^{2}$ A baixa escolaridade é um fator de risco devido à associação com precariedade na condição de vida e saúde. Um estudo, realizado na área rural da Índia, observou a relação do analfabetismo com as péssimas condições de vida de pessoas infectadas pelo bacilo de Hansen. ${ }^{18}$

Quanto à variável religião, foi identificado o catolicismo como maior número de adeptos. A religião interfere no processo saúde-doença por meio dos significados que o indivíduo traz do contexto espiritual, haja vista, que esse influencia em suas práticas, crenças e orientações quanto ao seu modo de vida e à sua saúde sexual reprodutiva. ${ }^{19}$

No que se refere às formas clínicas, a forma indeterminada apresentou baixo percentual entre os casos diagnosticados, denotando que a identificação das formas precoces ainda é precária. É importante ressaltar que, nesta fase, o diagnóstico adequado e o tratamento precoce interrompem a cadeia de transmissão. Destaca-se ainda, o percentual de casos sem classificação clínica. Interpreta-se, por meio dos dados, que os profissionais de saúde possuem dificuldades para classificar os casos segundo as formas clínicas no momento da assistência. ${ }^{18}$

Nesta premissa, verificou-se que a forma dimorfa foi a mais encontrada entre as mulheres avaliadas. Estas características são semelhantes às encontradas em estudos realizados em Minas Gerais e Fortaleza, os quais relataram a predominância de hanseníase no sexo feminino e as formas multibacilares. ${ }^{16-20}$ A OMS, em 1988, estabeleceu critérios clínicos, que consideram paucibacilares casos de pessoas com até cinco lesões cutâneas e/ou um tronco nervoso acometido, e multibacilares casos com mais de cinco lesões cutâneas e/ou mais de um tronco nervoso acometido. ${ }^{21}$

Em relação às baciloscopias, grande parcela das mulheres investigadas, não foi encaminhada à submissão deste exame. A baciloscopia da hanseníase é importante para o diagnóstico diferencial nos casos suspeitos de recidivas e na própria classificação da hanseníase, tendo em vista que a positividade do exame determina que o indivíduo acometido é classificado como multibacilar, responsável, por sua vez, pela transmissão. ${ }^{4}$ 


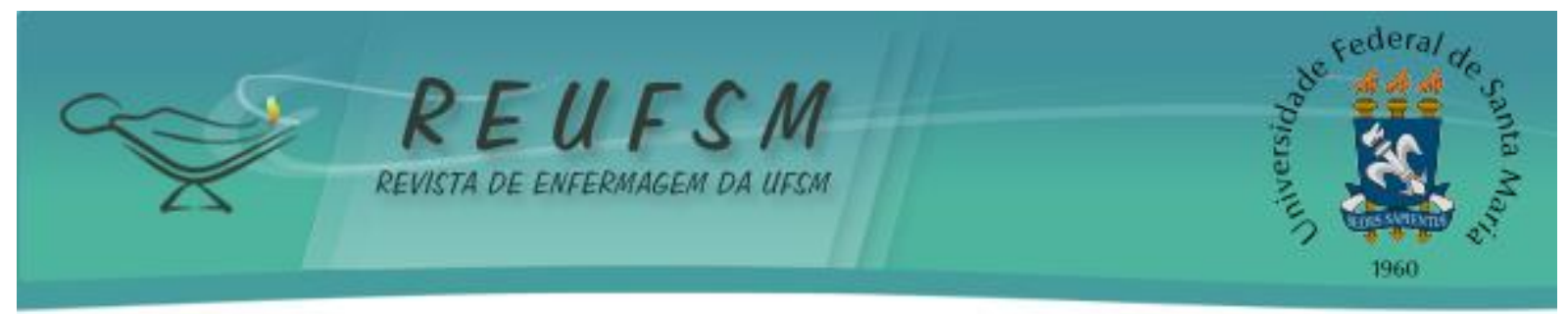

No que concerne à avaliação do grau de incapacidade física no momento do diagnóstico, ressalta-se um alto percentual de Grau Zero, o que significa que a maioria das mulheres não apresentava nenhum comprometimento neurológico nos olhos, mãos e pés. No entanto, foi perceptível um significativo índice de Grau I, revelando diagnóstico tardio. O grau de incapacidade física está diretamente relacionado ao tempo de evolução da doença, o que reforça a necessidade de estruturação de seguimento qualificado, na perspectiva da integralidade de todos os casos diagnosticados durante e após a poliquimioterapia. Os Graus I e II indicam o comprometimento de mecanismos de defesa devido ao processo do adoecimento, como sensação dolorosa, deixando, assim, o portador de hanseníase mais vulnerável a queimaduras, feridas, infecções, amputações, dentre outros. ${ }^{22-23}$

Diante desta problemática, as sequelas causadas pela hanseníase afetam diretamente a qualidade de vida destas mulheres, uma vez que essas terão dificuldade nas atividades da vida diária, no próprio sustento e de sua família, gerando repercussões de ordem psicológica, social e física, além de comprometer sua autoimagem feminina. ${ }^{24}$

Quanto à avaliação de incapacidades físicas no momento da alta, denota-se um alto percentual de mulheres que não foram submetidas a essa avaliação. Sabe-se, que as deformidades físicas causam estigma e isolamento social, com isso, é importante o monitoramento das alterações neurais para prevenção e controle das incapacidades, dependendo, tais ações, de um profissional de saúde qualificado durante o diagnóstico, tratamento e no momento da pós-alta. ${ }^{25}$

No que se refere ao modo de detecção, a maioria das mulheres procurou o serviço espontaneamente. De igual maneira, na Paraíba, a dominância na avaliação dos casos deu-se por encaminhamentos, demanda espontânea e outros modos, demonstrando negligência do serviço quanto à busca ativa de casos, comprometendo um possível diagnóstico precoce. ${ }^{1}$

Os dados mostraram precariedade entre os contatos registrados e examinados. $\mathrm{O}$ controle dos comunicantes é uma estratégia para redução da cadeia epidemiológica da hanseníase, bem como para o controle da infecção, devido ao risco da doença ser transmitida aos contatos domiciliares. Apesar disso, a avaliação dos contatos tem sido pouco valorizada e até mesmo negligenciada pelos serviços e profissionais de saúde. ${ }^{1-4}$

Neste cenário, convém mencionar que, mais da metade das mulheres avaliadas apresentaram episódios reacionais em algum momento de suas vidas (antes do diagnóstico, 


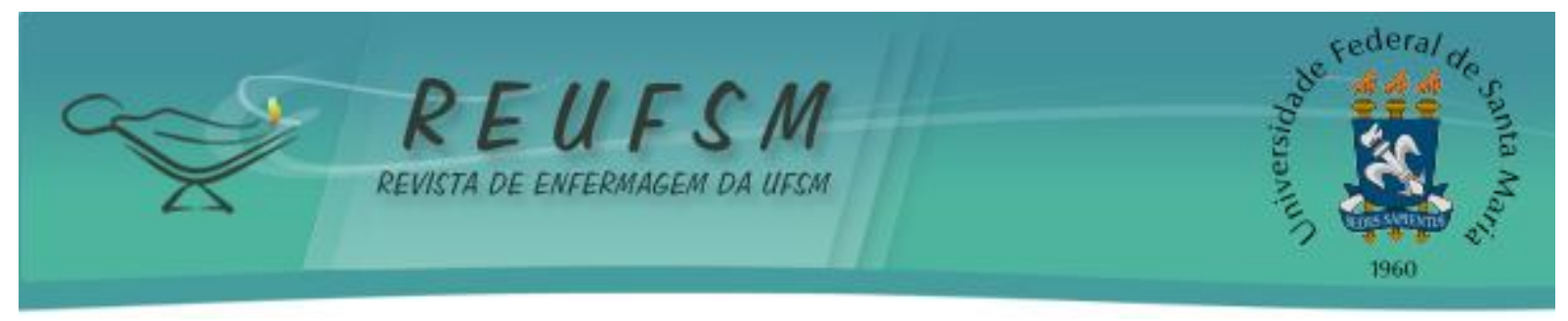

durante o tratamento e pós-tratamento). Fortalecendo este dado, uma pesquisa realizada no Centro de Referência para o Diagnóstico de Hanseníase e Tuberculose, localizado em Petrolina/Pernambuco, tratou que os fatores psicológicos, como medo, ansiedade e depressão, podem estar presentes em mulheres com hanseníase, pois, reduzem o sistema imunológico e assim, intensificam as reações. ${ }^{8}$

\section{CONCLUSÃO}

As características demográficas, sociais e clinicas da população estuda encontrada foram: mulheres economicamente ativas, predominantemente analfabetas e com ensino fundamental incompleto, que possuem companheiros, desempenham ocupações remuneradas e domésticas, compartilham o domicílio com seis ou mais familiares e, a maioria procuram a UBS espontaneamente ou por meio de encaminhamento.

Quanto aos aspectos clínicos da doença, merece destaque a forma dimorfa. É importante ressaltar, que boa parte dessas mulheres não foram submetida ao exame de baciloscopia. Realidade preocupante, tendo em vista, que a grande parcela das entrevistadas foram classificadas como multibacilares, e a realização do exame ajudaria na confirmação diagnóstica. Devido ao diagnóstico tardio, foi observado um alto índice de mulheres com comprometimento neural. Tais resultados, chama atenção para elaboração de ações dentro das UBS e na própria comunidade para orientar a população quanto aos sinais e sintomas da doença e suas complicações.

Como limitações deste estudo, verificou-se baixa cobertura da Estratégia Saúde da Família (ESF) no município de Maceió, desta forma, houve dificuldade com a localização dessas mulheres, por efeito disso, as visitas foram realizadas pelos próprios pesquisadores, sem a presença dos Agentes Comunitários de Saúde (ACS). Além de que, muitos endereços estavam desatualizados, consequentemente, muitas mulheres não foram encontradas.

Desta forma, o estudo possibilita uma reflexão sobre a importância da saúde da mulher, em idade reprodutiva acometida por doença infectocontagiosa. As informações aqui descritas poderão servir de base na elaboração de ações que visem a prevenção, diagnóstico, tratamento e cuidados pós-alta das pessoas atingidas pela hanseníase. Nesta perspectiva, sugere-se novas pesquisas que analisem as causas, consequências e influencias que dificultam 


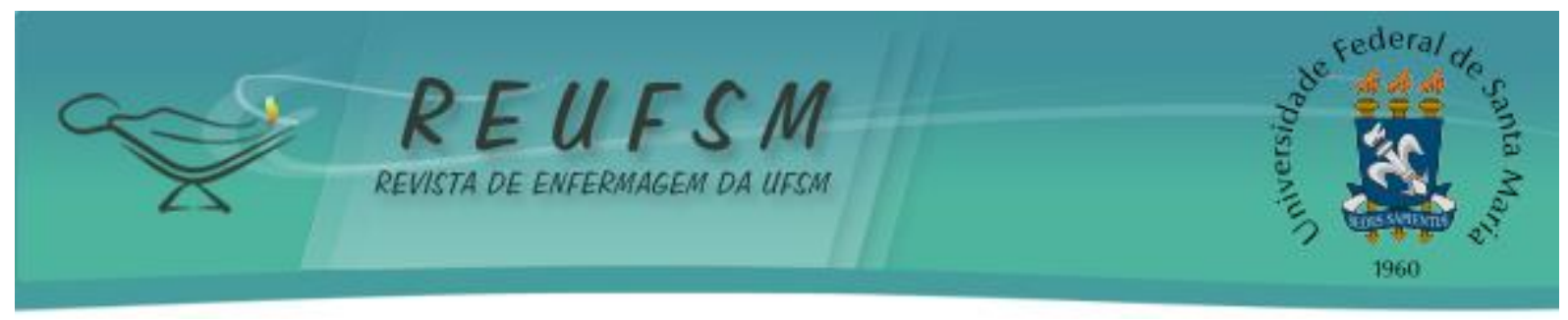

o monitoramento e assistência a estas mulheres, tendo em vista, que a grande parcela foram diagnosticadas na forma polarizada multibacilar e com incapacidades físicas.

\section{REFERÊNCIAS}

1. Brito KKG, Andrade SSC, Diniz IV, Matos SDO, Oliveira SHS, Oliveira MJGO. Caracterização dos casos de hanseníase diagnosticados através do exame de contato. Rev Enferm UFPE [Internet]. 2016 [acesso em 2017 maio 16];10(2):435-41. Disponível m: http://www.revista.ufpe.br/revistaenfermagem/index.php/revista/article/viewFile/8584/pdf_9529.

2. Gomes FC, Oliveira TC, Araújo JER, Félix LG, Araújo KMFA. Conhecimento do usuário da atenção primária à saúde acerca da hanseníase. Rev Enferm UFPE [Internet]. 2014 [acesso 2017 maio 16];8(8):3669-79. Disponível em: www.periodicos.ufpe.br/revistas/revistaenfermagem/article/download/10108/10580.

3. World Health Organization (WHO). Global leprosy update, 2013: reducing disease burden. Weekly Epidemiol Rec [Internet]. 2014 [acesso em 2017 maio 16];36(89): 389-400. Disponível em: http://www.who.int/wer/2014/wer8936.pdf.

4. Brasil. Ministério da Saúde. Diretrizes para vigilância, atenção e eliminação da Hanseníase como problema de saúde pública: manual técnico-operacional [Internet]. Brasília: Ministério da Saúde; 2016 [acesso em 2017 abr 21]. Disponível em: http://portal.saude.pe.gov.br/sites/portal.saude.pe.gov.br/files/diretrizes_para_._eliminacao_h anseniase_-_manual_-_3fev16_isbn_nucom_final_2.pdf.

5. World Health Organization (WHO).Global leprosy update, 2015: time for action, accountability and inclusion. Weekly Epidemiol Rec [Internet]. 2016 [acesso em 2017 jun 22]; 35(91):405-20.

Disponívelem: http://apps.who.int/iris/bitstream/10665/249601/1/WER9135.pdf?ua=1.

6. Brasil. Ministério da Saúde. Situação Epidemiológica. Taxa de detecção geral de casos novos de hanseníase, estados, Brasil, 2015 [Internet]. Brasília; Ministério da Saúde; 2016 [acesso em 2017 abr 21]. Disponível http://portalarquivos.saude.gov.br/images/pdf/2016/julho/07/Taxa-de-detec----o-geral-decasos-novos-de-hansen--ase--estados--Brasil--2015..pdf.

7. Kannambal K, Tharini GK. A screening study on dermatoses in pregnancy. J Clin Diagn Res [Internet]. 2017 [acesso em 2018 maio 06];11(5):WC01-WC05. Disponível em: https://www.ncbi.nlm.nih.gov/pmc/articles/PMC5483789/.

8. Santana LD, Silva SPC, Lira MOSC, Vieira MCA, Santos NTN, Silva TIM. Significado da doença para mulheres com hanseníase. Rev Enferm UFSM [Internet]. 2017 [acesso 2018 maio 02];7(1):111-22. https://periodicos.ufsm.br/reufsm/article/view/24824/pdf.

Disponivel em:

9. Ferreira LOC, Andrade AR, Santos TMF, Melo MCB, Rocha TTA. Prevalência de hanseníase em mulheres privadas de liberdade na região metropolitana do Recife em 2013. Rev Saúde Pesquisa [Internet]. 2016 [acesso em 2018 maio 01];9(2):227-33. Disponível em: http://periodicos.unicesumar.edu.br/index.php/saudpesq/article/view/4993/2834. 


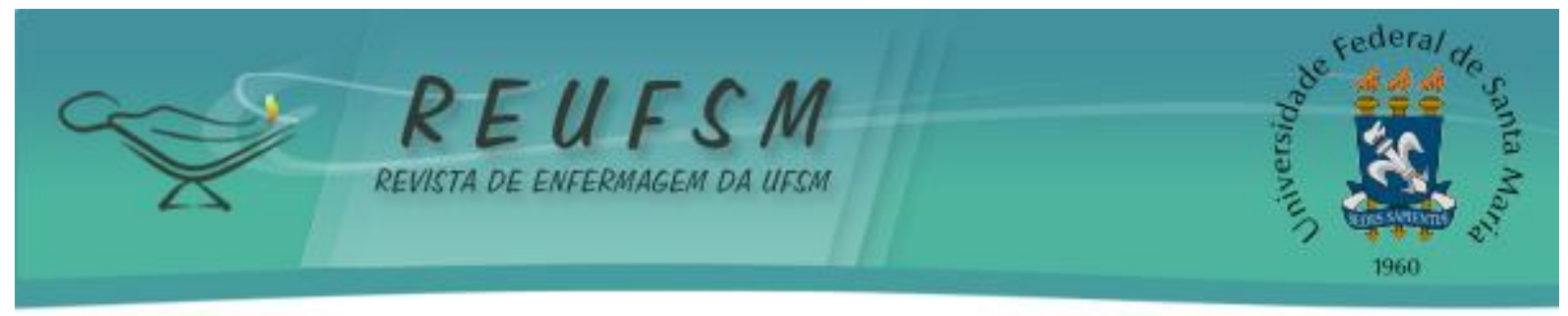

10. Monte, RS; Pereiraz MLD. Hansen's disease: social representations of affected people. Rev Rene [Internet]. 2015 [acesso 2018 maio 01];16(6):863-71. Disponível em: http://www.revistarene.ufc.br/revista/index.php/revista/article/view/2221/pdf_1.

11. Loose JTT, Diniz SN, Batista EC, Carlotto MS, Cunha DF, Ferreira DF. Qualidade de vida em mulheres com episódios reacionais hansênicos em uso de prednisona no município de Rolim de Moura - RO. Rev Ciên Saúde [Internet]. 2017 [acesso em 2018 maio 01];2(2):1-10. Disponível em: http://revistaeletronicafunvic.org/index.php/c14ffd10/article/view/70/69.

12. Carvalho RKAL, Tavares CM, Silva JMO, Holanda JBL. Ciclogravídico puerperal associado às reações hansênicas: um olhar pela enfermagem. Rev Enferm UFPE [Internet]. 2015 [acesso em 2017 jul 26];9(4):7272-80. Disponívelem: https://periodicos.ufpe.br/revistas/revistaenfermagem/article/view/13584/1639.

13. Gonçalves M, Prado AR, Silva SS, Santos KS, Araujo PN, Fortuna CM. Work and leprosy: women in their pains, struggles and toils. Rev Bras Enferm [Internet]. 2018 [acesso em 2018 maio 01];71(Supl 1):660-7. Disponível em: http://www.scielo.br/pdf/reben/v71s1/0034-7167-reben-71-s1-0660.pdf.

14. Lopes VAS, Rangel EM. Hanseníase e vulnerabilidade social: uma análise do perfil socioeconômico de usuários em tratamento irregular. Saúde Debate [Internet]. 2014 [acesso em 2017 jul 21];38(103):817-29. Disponível em: http://www.scielo.br/pdf/sdeb/v38n103/0103-1104-sdeb-38-103-0817.pdf.

15. Brito KKG, Araújo DAL, Uchôa REMN, Ferreira JDL, Soares MJGO, Lima JO. Epidemiologia da hanseníase em um estado do nordeste brasileiro. Rev Enferm UFPE [Internet]. 2014 [acesso em 2017 abr 20]; 8(8):2686-93. Disponívelem: http://www.revista.ufpe.br/revistaenfermagem/index.php/revista/article/viewFile/6092/pdf_5872.

16. Melo JP, Moraes MM, Santos NR, Santos TS. Perfil epidemiológico os casos de hanseníse de uma unidade de saúde. Rev Saude Colet UEFS [Internet]. 2017 [acesso em 2018 maio 06];7(1):29-34. Disponível em: http://periodicos.uefs.br/index.php/saudecoletiva/article/view/1176/1279.

17. Fontes ANB, Lima LNGC, Mota RMS, Almeida RLF, Pontes MA, Gonçalves HS, et al. Genotyping of Mycobacterium leprae for better understanding of leprosy transmission in Fortaleza, Northeastern Brazil. Plos Negl Trop Dis [Internet]. 2017 [acesso em 2018 maio 06];11(12):e0006117. Disponível em: http://journals.plos.org/plosntds/article/file?id=10.1371/journal.pntd.0006117\&type=printable.

18. Silva MS, Silva EP, Monteiro FF, Teles SF. Perfil clínico-epidemiológico da hanseníase no estado do Acre: estudo retrospectivo. Hansen Int [Internet]. 2014 [acesso em 2017 abr 30]; 39(2):19-26. Disponível em: http://www.ilsl.br/revista/detalhe_artigo.php?id=12345.

19. Coutinho RZ, Ribeiro PM. Religião, religiosidade e iniciação sexual na adolescência e juventude: lições de uma revisão bibliográfica sistemática de mais de meio século de pesquisas. Rev Bras Est Pop [Internet]. 2014 [acesso em 2018 maio 02];31(2):333-65. Disponível em: http://www.scielo.br/pdf/rbepop/v31n2/a06v31n2.pdf.

20. Souza VB, Silva MRF, Silva LMS, Torres RAM, Gomes KWL, Fernandes MC, et al. Perfil epidemiológico dos casos de hanseníase de um centro de saúde da família. Rev Bras 


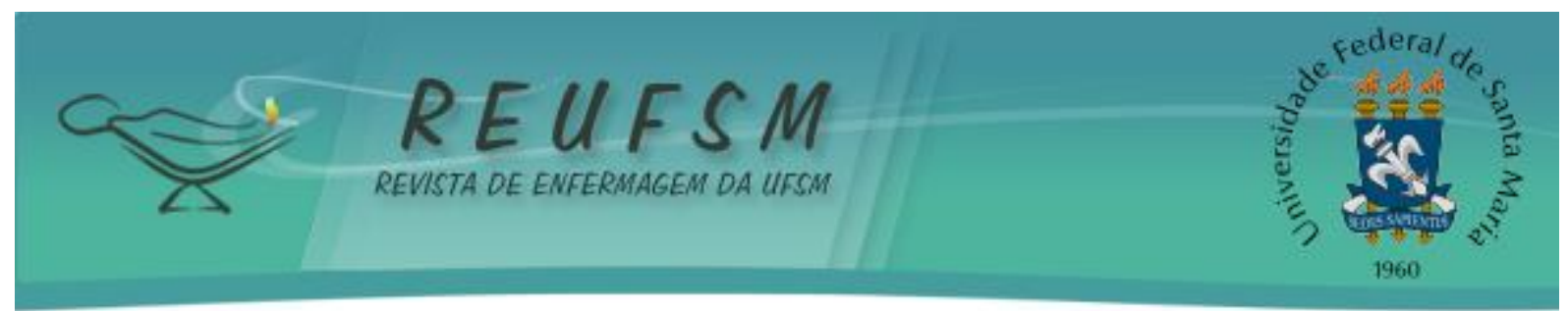

Promoção Saúde [Internet]. 2013 [acesso em 2017 jul 23 ];26(1):110-6. Disponível em:http://www.unifor.br/images/pdfs/rbps/2013.1_artigo14.pdf.

21. Lastória JC, Abreu MAMM. Leprosy: review of the epidemiological, clinical, and etiopathogenic aspects - Part 1. An Bras Dermatol [Internet]. 2014 [acesso em 2017 abr 30]; 89(2):205-18.

Disponível

em:

https://www.ncbi.nlm.nih.gov/pmc/articles/PMC4008049/pdf/abd-89-02-0205.pdf.

22. Ribeiro GC, Fabri ACOC, Amaral EP, Machado IE, Lana FCF. Estimativa da prevalência oculta da hanseníase na microrregião de Diamantina - Minas Gerais. Rev Eletr Enferm [Internet]. 2014 [acesso em 2017 abr 30]; 16(4):728-35. Disponível em: https://www.fen.ufg.br/fen_revista/v16/n4/pdf/v16n4a04.pdf.

23. Brito KKG, Andrade SSC, Santana EMF, Peixoto VB, Nogueira JA, Soares MJGO. Epidemiological analysis of leprosy in an endemic state of northeastern Brazil. Rev Gaúch Enferm [Internet]. 2015 [acesso em 2017 abr 30];36(N Esp):24-30. Disponível em: http://www.scielo.br/pdf/rgenf/v36nspe/en_0102-6933-rgenf-36-spe-0024.pdf.

24. Santana LD, Silva SPC, Lira MOSC, Vieira MCA, Santos NTN, Siva TIM. Significado da doença para mulheres com hanseníase. Rev Enferm UFSM [Internet]. 2017 [acesso em 2017 jul 28];7(1):111-22. Disponível em: https://periodicos.ufsm.br/reufsm/article/view/24824/pdf.

25. Rosa GR, Lima MM, Brito WI, Moreira AM. Análise da completude de incapacidade em hanseníase da regional de saúde de Rondonópolis/MT. Rev Eletr Gestão \& Saúde [Internet]. 2016 [acesso em 2017 abr 30];7(1):82-95. Disponível em: https://dialnet.unirioja.es/descarga/articulo/5555860.pdf.

Data de submissão: 29/11/2017

Data de aceite: 28/05/2018

Autor correspondente: Clodis Maria Tavares

E-mail: clodistavares@yahoo.com.br

Endereço: Rua Santa Fernanda, nº 641, Jatiúca. Maceió/Alagoas, Brasil.

CEP: 57035-670 